\title{
Metabolomics: Where seeing is believing
}

Pop quiz: What's the best molecular indicator of the status of a cell? It isn't the genome, which remains more or less unchanged during growth, development, and disease. Nor is it the proteome or the transcriptome; though protein abundance and mRNA expression do serve as molecular bellwethers of the cellular condition, these biomolecules turn over far too slowly.

When it comes to cellular status, the molecular canary-in-the-mineshaft that researchers should look to is small molecules-or metabolites-says Renato Zenobi, professor of chemistry and applied biosciences at ETH Zurich. "The metabolome is arguably the most sensitive measure of a cellular phenotype," he and Matthias Heinemann of the University of Groningen, the Netherlands, wrote in 2010 (1). Whereas proteins and mRNA turn over in minutes to hours, Zenobi says, the metabolome "changes on the time scale of seconds."

To measure those changes, researchers typically use nuclear magnetic resonance (NMR) or mass spectrometry (MS), both of which, generally speaking, assess the bulk abundance of metabolites in populations of cells. Increasingly, though, such population-based approaches are proving unsatisfactory.

Jonathan Sweedler, the James R. Eiszner Family Chair in Chemistry at the University of Illinois, Urbana-Champaign, studies neurotransmitters and neuropeptides. According to him, some neurons express molecules at high abundance, while others don't express them at all. Homogenizing brain tissue and analyzing it directly would produce data representing an average over all the cells in a sample. Interrogating cells one by one, on the other hand, leads to a very different picture.

Today, Sweedler can focus on those individual cells, thanks to a technique called MS imaging (MSI) that couples the exquisite sensitivity and mass accuracy of MS with the cellular (or nearly cellular) spatial resolution of imaging. MSI is not without issues; not all molecules ionize equally well, for instance, and mass spectrometers struggle to capture the less-abundant molecules in a sample. But the approach is quickly evolving, and researchers can now apply MSI to living

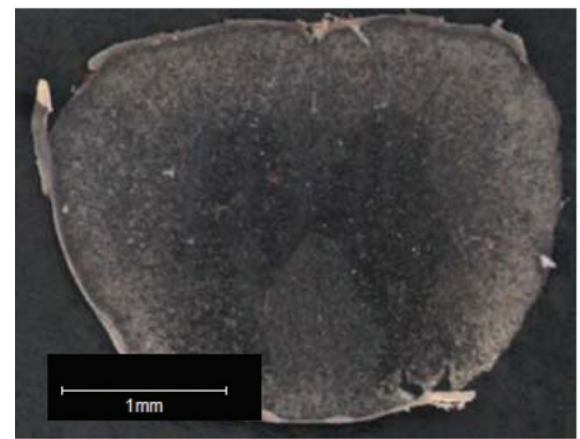

Optical image

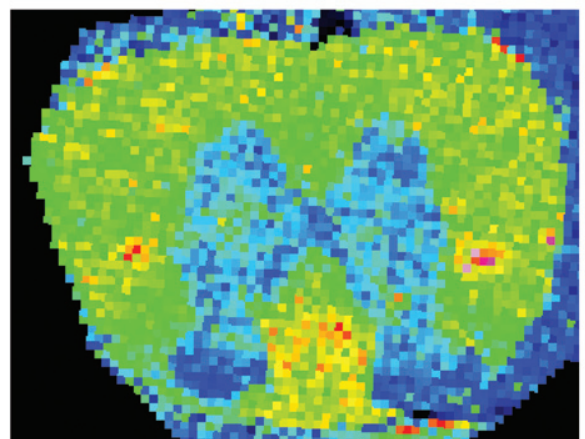

Mass spectrometry imaging of a transverse section of the rat spinal cord reveals unique molecular distributions. Here two lipids (phosphatidylcholine 42:4 and phosphatidylethanolamine 38:2) are localized in the peripheral white matter and dorsal horns respectively, and a third unidentified ion shows an interesting distribution as well. Image courtesy of E.J. Lanni , S.S. Rubakhin, and J.V. Sweedler, University of Illinois at Urbana-Champaign.

samples in the lab or clinic, as well as in the 3-D molecular reconstruction of biological samples, among other things.

\section{MALDI MSI}

While several forms of MSI exist, all share certain key features: A tissue sample is repeatedly interrogated by an MS ionization source point by point, resulting in a multidimensional dataset in which each $x-y$ coordinate (or pixel) is associated with a mass spectrum. Those spectra may themselves contain hundreds or even thousands of peaks, each of which can be represented graphically by a distinct color.

Richard Caprioli, the Stanley Cohen Professor of Biochemistry at the Vanderbilt University School of Medicine, likens this kind of representation to the color channels in a digital image. Just as every pixel in a digital picture can be expressed in terms of red, green, and blue channels, biological

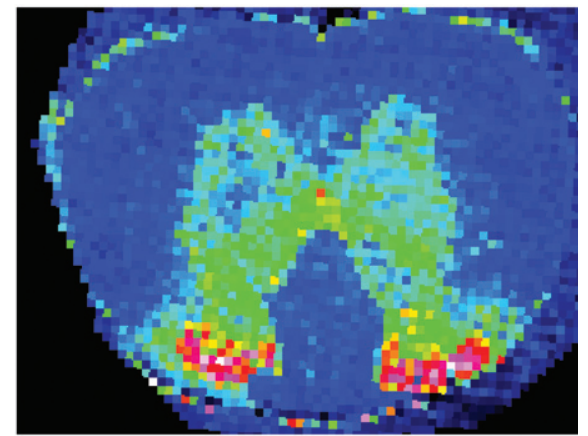

$\mathrm{m} / \mathrm{z} 772.7$ - PE $38: 2[\mathrm{M}+\mathrm{H}]^{+}$

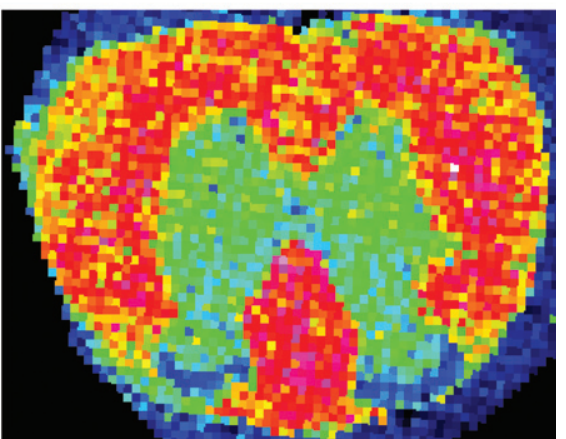

pixels in an MSI experiment can be represented in terms of discrete molecular masses.

"Let's say you are looking at a piece of tissue and there are hundreds or thousands of compounds that you can ablate with the laser," Caprioli explains. "We can take a whole spectrum and pick out one signal and ask how that signal changes over the thousands of pixels that we make in the image."

Of course, the technique isn't limited to a single channel; MSI data can theoretically contain thousands of discrete channels. Caprioli's team is working to identify multi-component molecular signatures of disease-collections of six or eight MS peaks whose presence or absence correlates with disease progression and prognosis. His team has already published signatures for lung, skin, and brain tumors, and is now turning its attention to prostate cancer.

Caprioli favors MALDI-based MSI, in which a tissue section is placed on a MALDI 


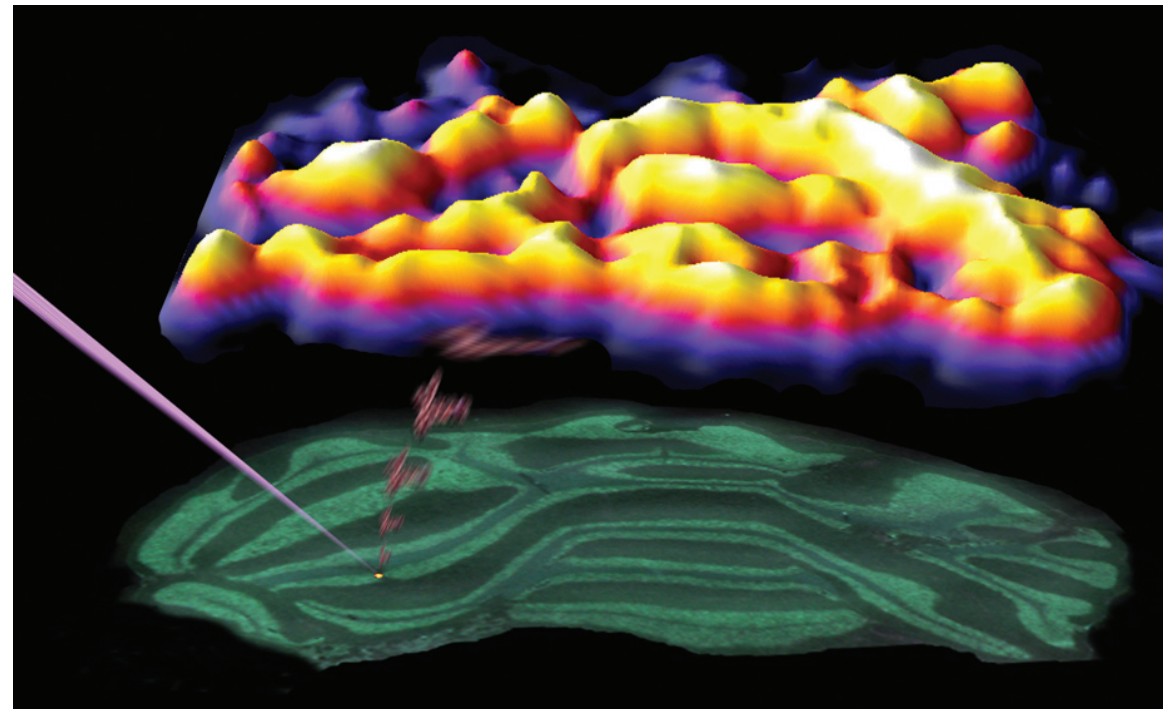

Imaging metabolites from brain tissue using nanostructure-initiator mass spectrometry (NIMS) technology. Courtesy of Gary J. Patti, Jon V. Apon and Gary Siuzdak, Scripps Research Institute.

target plate, coated with a matrix material, and placed inside the mass spectrometer under vacuum. There, an ultraviolet laser raster is performed across the sample, collecting spectra at each user-defined pixel.

Two variables govern resolution in MALDI-MS: how the matrix is deposited on the sample, and the spot size of the MALDI laser. Several years ago, Caprioli's team was imaging with 50- to 80-micron pixel resolution (five cell diameters); now he says they routinely focus down to 20 microns, and sometimes even attain 2 microns (subcellular).

But smaller isn't always better. For one thing, smaller pixels means more spectra per unit area, which increases both data acquisition time and dataset size. A typical liquid chromatography-MS run produces datasets between 20 and $40 \mathrm{MB}$ in size, according to Lawrence Berkeley National Lab staff scientist Trent Northen, who used an alternative technique called nanostructureinitiator MS (NIMS) to acquire 3-D molecular images of mouse breast tumors. "When we image a tumor, we might have 40 gigabytes of data per section," he says. Those data have to be stored, compared, and analyzed if researchers are to distill biological insights, an effort whose complexity scales with decreasing pixel size. Caprioli's lab has filled some $50 \mathrm{~TB}$ of hard disk space and shows no signs of slowing down. "The way we're going," he says, "it will be six or eight months before we get the next 50 ."

Smaller laser points mean more data, but also less ionized material per shot. "Sample volume goes with the cube of the spot size," explains Akos Vertes, co-director of the W.M. Keck Institute for Proteomics Technology and Applications at George Washington University in Washington, DC, who uses another MSI technique called laser ablation electrospray ionization (LAESI) for his research. A four-fold reduction in spot diameter (from 80 to 20 microns) yields 64-fold fewer ions.

"In imaging mass spectrometry, it's always a tradeoff between spatial resolution and chemical information," says Sweedler. It's like comparing a powerful telescope with a very narrow field of view and weaker binoculars with a wider field of view. "If you're looking at the feather on a bird sitting in a tree, you want that very narrow field and high power," Caprioli says. "But if you're looking at a football game sitting in the stands, you won't see anything with the high power; you want a wide-scope binocular." Lu, Penn State University.

\section{Matrix Matters}

In MALDI, the matrix absorbs laser energy, channeling it to both vaporize and ionize the sample. But matrix is also the technique's biggest shortcoming.

For one thing, matrix application isn't trivial. Matrices work by extracting biological material from the underlying tissue and co-crystallizing it into a more ionizable form. For that to work, the matrix must be uniformly sprayed on the tissue with either a robot or an airbrush, which is an expensive and time-consuming process. The matrix can also redistribute cellular contents, which blurs cellular boundaries, and produces fragment ions on the low-molecular weight end of mass spectra, confusing metabolomic experiments. Many researchers have thus developed strategies that avoid matrix.

NIMS, for instance, trades matrix for a porous silicon base topped with a Teflonlike polymer and the tissue sample. Irradiating the target with a MALDI UV laser heats the silicon, which vaporizes the polymer and desorbs and ionizes the tissue. "The polymer itself doesn't ionize very well, minimizing background and making the surface very useful for analyzing smallmolecule metabolites," explains Gary Siuzdak, director of the Center for Metabolomics and Mass Spectrometry at the Scripps Research Institute in La Jolla, CA, whose lab developed NIMS.

Siuzdak has used NIMS to study the distribution of cholesterol and cholesterol precursors in brain slices from a mouse model of Smith-Lemli-Opitz syndrome, a genetic condition causing errant cholesterol biosynthesis and subsequent severe mental retardation. Normally, says Siuzdak, sterols don't ionize efficiently, but his team

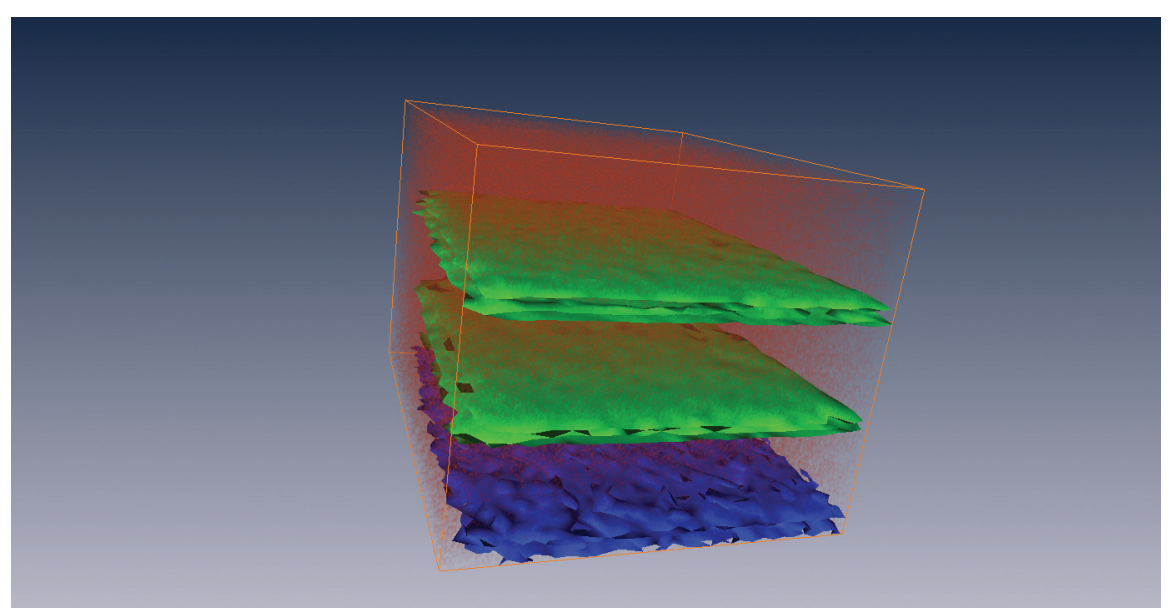

Three-dimensional C60 TOF-SIMS image of two lipid bilayers (green) embedded into a second lipid matrix (red) deposited onto silicon (blue). The film is $\sim 100 \mathrm{~nm}$ thick, and the bilayers are $\sim 4 \mathrm{~nm}$ thick. Drawing courtesy of Nicholas Winograd and Caiyan 


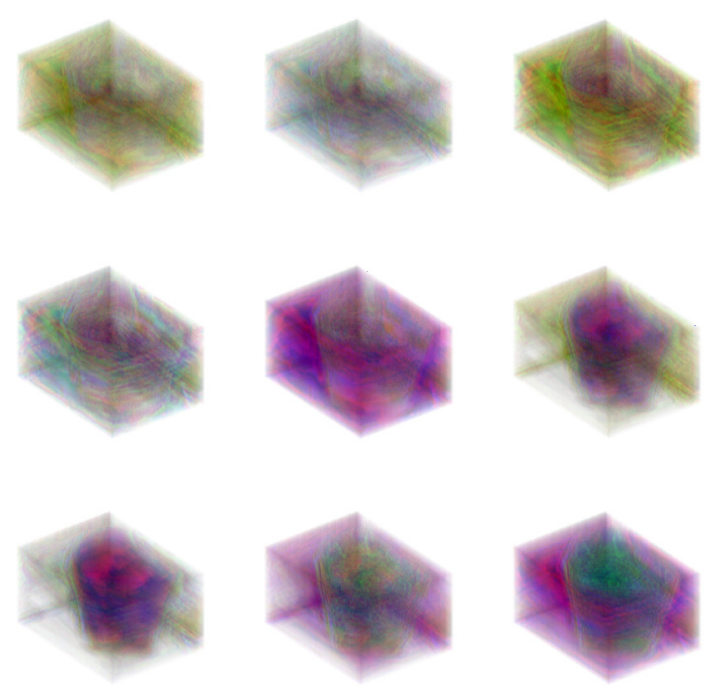

Molecular profile of a tumor, in 3-D. Trent Northen and Ben Bowen imaged serial sections of a mouse mammary tumor using NIMS, and then used automatic alignment of the sections to produce a 3-D model for each $\mathrm{m} / \mathrm{z}$ value. To identify regions with different metabolite distributions, nonnegative matrix factorization was used, where each region is represented by a color. All unique combinations of the regions are displayed. Image courtesy of Ben Bowen, Berkeley Lab.

coats the NIMS polymer with silver, which makes the cholesterol more ionizable.

Siuzdak's team has developed 8 or 10 distinct surfaces, each designed to enhance ionization of a particular molecular class. Such tricks help overcome one of MSI's key limitations, he says. In standard MS, the input sample is generally subjected to purification and separation techniques that tend to reduce ion competition, enabling researchers to see thousands of distinct molecular species. However, since the sample is ionized directly in MSI, says Siuzdak, "we are typically limited by the most dominant molecules or the ones that ionize best."

Ales Svatos, group leader of the Mass Spectrometry Research Group at the Max Planck Institute for Chemical Ecology, takes a different approach, exploiting the UV laser's ability to specifically excite UV-absorbing compounds. "In plant tissue, there are a lot of such compounds," he notes, "and they are of general interest, like phenolic compounds and flavonoids." In one study, Svatos' team used this technique (called matrix-free laser desorption/ionization MSI, or LDI-MSI) to localize pharmacologic compounds called hypericins to secretory "cavities" on the leaves of St. Johns wort, with single-cell ( $<10$-micron) resolution.

R. Graham Cooks, professor of chemistry at Purdue University (West Lafayette, IN), divides MSI techniques into two broad classes: those that require the sample to be analyzed in a vacuum, and those that do not. MALDI, NIMS, and LDI-MSI fall into the former category. But desorption electrospray ionization (DESI), a matrix-free technique developed by Cook, is a so-called "ambient ionization method," meaning it occurs in ambient air and requires minimal or even no sample processing. "Ambient ionizationbecause of the lack of work-up of the sample, and because the sample is outside the mass spectrometer-is characterized by high throughput," he says.

In DESI, fine droplets of about 5 microns in size are directed at a tissue sample in air. As they collect on the surface, these droplets form a puddle that extracts small molecules from the underlying

\section{Any gene. Any locus. Any cell line.}

Endogenous gene targeting using rAAV vectors

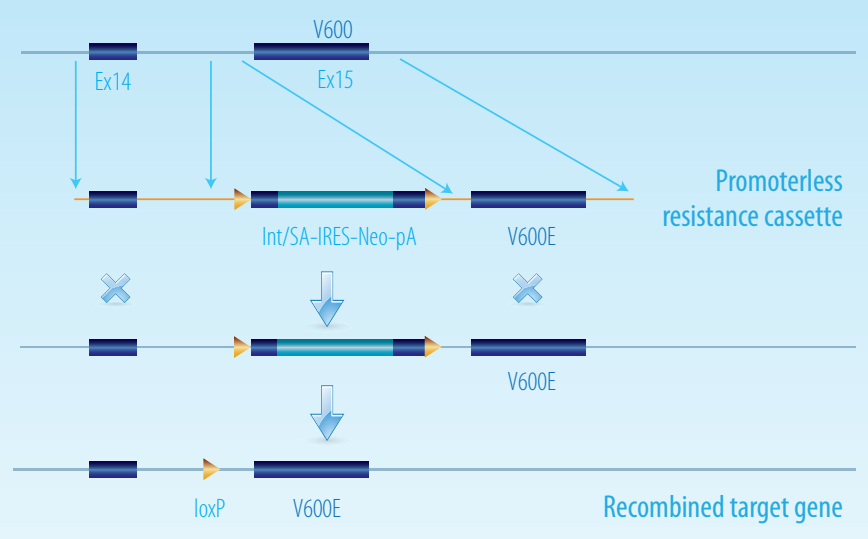

\section{Vector Features}

Recombinant adeno-associated virus (rAAV) vector

Single-stranded genome, stimulates endogenous HR

No double-strand DNA breaks, zero off-target effects

\section{Applications}

Target any mammalian cell type

Gene knock-in: Mutations, SNPs, Protein tag insertions

Gene knock-out: Mutations, Optimized bioproducer lines

\section{Available Now}

Cell lines

Over 300 ready-to-ship X-MAN (Mutant And Normal) human isogenic cell line pairs

\section{Services}

Custom mammalian cell line modification, compound screening, standard \& bespoke assays

\section{Reagents}

Gold-standard genetically-defined genomic DNA reagents

\author{
X-MAN 2011 Catalogue new \\ To receive your free copy email brochure@horizondiscovery.com
}

For more information on rAAV genome editing and an extensive reference library, visit www. horizondiscovery.com 


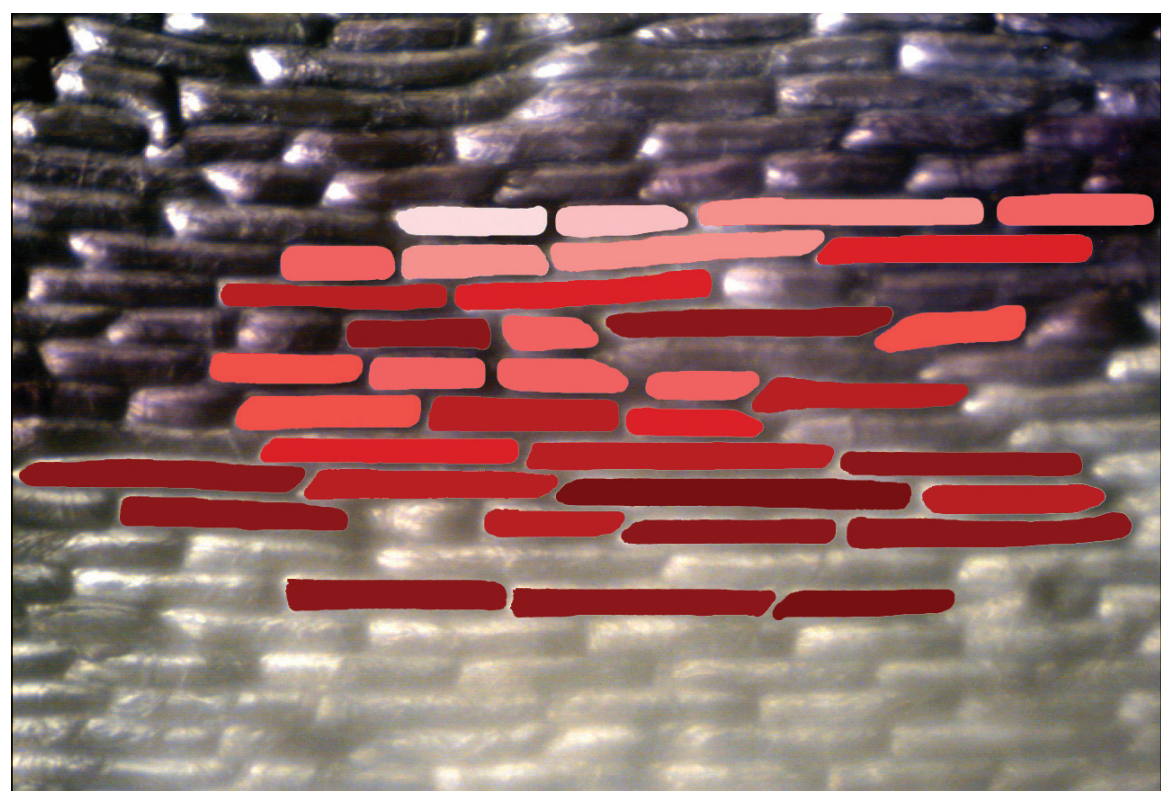

Cell-by-cell imaging of quercetin glucoside distribution in epidermal cells of an onion (Allium cepa) bulb at the transition from nonpigmented to pigmented tissue boundary (lighter colors indicate higher ion concentrations). Courtesy of Bindesh Shrestha.

tissue. Subsequent droplets cause that solution to then splash up into the instrument's inlet, where they are analyzed. In one recent study, Cooks' team used DESI to grade human brain tumor specimens based entirely on their lipid content.

According to Cooks, the lateral resolution of DESI can be as high as 50 microns. "[But] a lot of what we've published has been at 180 microns," he says. Still, for his ultimate application-guiding surgeons in tumor excision-that's just fine.

\section{3-D MSI}

Caprioli compares MSI to digital photography. But there's one variant that is more akin to confocal microscopy, in which tissue slices are optically sectioned as they are imaged. 3-D imaging is an MSI variant that couples MSI with a way to burrow into the sample at each pixel, producing what Nicholas Winograd, Evan Pugh Professor of Chemistry at Penn State University (University Park, PA), calls a "molecular depth profile."

Winograd employs secondary ion MS (SIMS), in which he bombards a sample with ionized 60-carbon buckyballsa collision Winograd likens to a meteor impact.

The technique requires no matrix, but it isn't ambient; SIMS runs in a vacuum. Each buckyball impact produces a crater some $2 \mathrm{~nm}$ in diameter and 5-10 $\mathrm{nm}$ deep, he says. The final lateral resolution is a couple of hundred nanometers - two orders of magnitude better than MALDI MSI, he says.
"The beauty," Winograd says, "is that you can remove material with nanometer precision and not do a great deal of chemical insult to the underlying material. You can almost remove organic material layer by layer and see what's underneath." In one experiment, his team imaged a 4-nm lipid bilayer buried beneath a lipid matrix, coupling the data with atomic force microscopy to view the topographic landscape as well. "It's very clean," he says of the data. "I'm blown away by it."

Vertes's LAESI offers an alternative approach. An ambient and matrix-free energy to excite the water naturally found in biological specimens. Irradiation with the mid-IR laser produces a plume of ions as well as nonionized vaporized material called neutrals. The latter far outnumber the former- "we are talking about $10^{-4}$ ion yields," he says - and normally, those neutrals are lost. But LAESI captures them in an electrospray plume, thereby increasing the technique's sensitivity. technique, LAESI uses mid-IR laser
As in SIMS, each LAESI laser strike drills down into a specimen. In one elegant proof-of-principle experiment, the team marked either side of a peace lily leaf with two different dyes in distinctive shapes. They then imaged the leaf at 3432 points (300-micron diameter) at each of six depths (30-40 microns deep), localizing the two dyes, as well as pigments such as chlorophyll, within the leaf structure.

Vertes has now reduced his LAESI focus size to 30 microns, about the size of a plant cell. At that diameter, he says, he can rethink his experimental approach, using the cells themselves as pixels. "This is a radically different way of building MS images," he says. "This way, we don't force an artificial rectangular grid over tissue that has a natural grid based on its cellular structure."

Vertes' team recently applied a 2-D cell-aspixel approach to profile sugar and pigment abundance in pigmented and nonpigmented onion epidermal cells. In graphical representation, these images resemble a wall of irregular stone, half-painted in shades of red, white, and pink - a heat map of metabolite abundance plotted cell-by-cell.

Unsurprisingly, the team found that pigmented cells contain more pigment than unpigmented cells. But the promise of the technique is greater than that. Caprioli says the real power of MSI is that it plays to a particular human strength: interpreting visual information. "We are built to process images and get lots of information from it," he says. MSI, he continues, "brings to science that dimension in a molecular way."

\section{References}

1. Heinemann, M. and R.Zenobi. 2010. Single cell metabolomics, Curr. Opin. Biotechnol. 22:1-6.

Written by Jeffrey M. Perkel, Ph.D. 四

BioTechniques 50:285-289 (May 2011)

doi 10.2144/000113664

To order reprints of this article, contact:

biotechniques@fosterprinting.com

\section{Custom Medica and Reagent Manufacturing}

Fast turn around time - ISO certified facility 30 years experience - Nationwide distribution

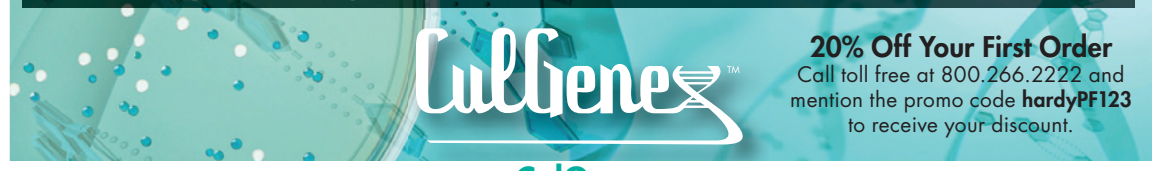

www.CulGenex.com 